\title{
Identification of ANXA1 as a Lymphatic Metastasis and Poor Prognostic Factor in Pancreatic Ductal Adenocarcinoma
}

\author{
Qing-Hua Liu' ${ }^{1,2}$, Mei-Lin Shi ${ }^{1,3}$, Jin Bai ${ }^{1 *}$, Jun-Nian Zheng ${ }^{1 *}$
}

\begin{abstract}
Objective: The aim of this study was to investigate the clinical significance of annexin a1 (ANXA1) and provide molecular evidence to support that decreased ANXA1 expression could enhance cancer migration and invasion in pancreatic ductal adenocarcinoma (PDAC). Materials and Methods: Immunohistochemistry of a tissue microarray with 162 surgically resected PDAC specimens was performed to examine the expression of ANXA1. We also investigated the relationship between ANXA1 expression and clinicopathological factors and prognosis of PDAC patients. We further studied the role of ANXA1 in PDAC cell proliferation, migration and invasion by cell proliferation assay, migration assay and matrigel invasion assay with reduced ANXA1 expression by RNAi. Western blotting was used to detect matrix metalloproteinase-9 (MMP-9), and tissue inhibitor of metalloproteinase-1 (TIMP-1) expression. We also detected MMP-9 enzyme activity by gelatin zymography. Results: Decreased expression of ANXA1 was significantly associated with poor differentiation, lymph node metastasis and advanced TNM stage of PDAC patients $(p<0.05)$. Moreover, decreased expression of ANXA1 was correlated with poor survival $(p<0.05)$. Furthermore, we found that ANXA1 knockdown inhibited cell proliferation, induced G1 phase cell cycle arrest, increased PDAC cell migration and invasion capacity compared with controls. In addition, Western blotting showed that ANXA1 knockdown increased the MMP-9 protein level and decreased TIMP-1 expression. Gelatin zymography showed that MMP-9 enzyme activity was also elevated. Conclusions: Negative ANXA1 expression is a most unfavorable prognostic factor for PDAC patients. ANXA1 knockdown inhibits cell proliferation by inducing G1 phase cell cycle arrest and increases migration and invasion of PDAC cells through up-regulating MMP-9 expression and activity, implying that ANXA1 may serve as a promising prognostic biomarker and therapeutic target for PDAC.
\end{abstract}

Keywords: Annexin a1 - pancreatic ductual adenocarcinoma - tissue microarray - lymphatic metastasis - prognostic

Asian Pac J Cancer Prev, 16 (7), 2719-2724

\section{Introduction}

PDAC is the fourth leading cause of cancer death per year in the United States and eighth worldwide with an expected incidence of 43,920 cases and 37,390 cases of death in 2012 (Siegel et al., 2012). The high mortality rates in PDAC patients are associated with metastatic disease, which may contribute to more than $75 \%$ of patients (Keleg et al., 2003). Metastasis is the result of several sequential steps of tumor development (Hynes, 2003). The factors driving this process are thus important for future therapeutic of tumor patients.

ANXA1 (also known as lipocortin-1) was the first member of the annexin superfamily of proteins reported in 1979, that was able to bind phospholipids in a calcium dependent manner (Flower and Blackwell, 1979). Although the exact function of ANXA1 remains unclear, it has been suggested that ANXA1 is associated with various physiological processes, furthermore, deregulation of ANXA1 correlates with tumor metastasis in several types of cancer, such as cholangiocarcinoma, nasopharyngeal carcinoma, colon cancer, et al (Hongsrichan et al., 2013; Li et al., 2013; Zeng et al., 2013). Up to now, the possible function of ANXA1 in the development, metastasis and prognostic of PDAC remains unclear and opinions differ on its precise role.

In the present study, we evaluated ANXA1 expression in 162 PDAC tissues and analyzed the correlation between ANXA1 expression and clinicopathological variables. Our data demonstrated that decreased expression of ANXA1 was associated with poor post-surgical survival, poor differentiation, lymphatic metastasis and advanced TNM stage of PDAC patients. In addition, we found that decreased expression of ANXA1 significantly increases PDAC cell migration and invasion by up-regulating MMP9 expression and activity. These data implied that ANXA1 may be used as a potential therapeutic target for PDAC.

\section{Materials and Methods}

Patients and samples

Samples of PDAC and tumor adjacent pancreatic

${ }^{1}$ Jiangsu Center for the Collaboration and Innovation of Cancer Biotherapy, Cancer Institute, ${ }^{2}$ Department of Pathology, ${ }^{3}$ School of Medical Imaging, Xuzhou Medical College, Xuzhou, China*For correspondence: bj@xzmc.edu.cn 
tissues were obtained from 113 patients who underwent surgical resection of pancreatic adenocarcinoma in Changhai Hospital, Secondary Military Medical University between 2002 and 2004, and 49 patients in the Affiliated Hospital of Xuzhou Medical College between 2005 and 2012 with informed consent under the guideline of Hospital Ethics Committee. The patients comprised 98 males and 64 females aged from 21 to 83 years old when they accepted surgery. None of these patients received any anti-cancer treatment before surgery. Correlation between the expression of ANXA1 and clinicopathological parameters including age, gender, tumor differentiation, lymph node metastases, nerve infiltration, clinical stage and prognosis were evaluated.

All patients have complete clinical information. Among them, 65 patients had follow-up until their death or until their most recent contact (April 3,2013). At the time of the most recent contact, 6 of 65 patients were still alive, whereas 59 patients were dead. This study was approved by the ethical committee of the Institutional Review Boards of Affiliated Hospital of Xuzhou Medical College and all examinations were performed after obtaining written informed consents.

\section{Immunohistochemistry}

Tissue microarrays were constructed using the 162 paraffin-embedded tumor tissue specimens by a precision arraying instrument (Beecher Instruments, Silver Spring, MD, USA). The sections from the tissue microarray blocks were deparaffinized, rehydrated and then heated in 0.01 $\mathrm{mol} / \mathrm{l}$ sodium citrate buffer (PH 6.0) for $8 \mathrm{~min}$ at $95^{\circ} \mathrm{C}$. Following incubation with $0.3 \%$ hydrogen peroxide in methanol for $15 \mathrm{~min}$ at room temperature and treatment with normal goat serum (Beijing Sequoia Jinqiao Biological Technology Co., Ltd.). Blocking serum was drained off and the slides were incubated overnight at $4^{\circ} \mathrm{C}$ with mouse anti-ANXA1 polyclonal antibody (TA500967, OriGene Technologies, Inc., USA) at a dilution of 1:75. Slides were rinsed for $10 \mathrm{~min}$ in PBS wash solution and incubated for $30 \mathrm{~min}$ with the HRP-labeled polymer conjugated secondary antibody (12127A07, Beijing Sequoia Jinqiao Biological Technology Co., Ltd.) was applied according to the manufacturer's instructions. A known positive endometrial cancer tissue biopsy sample was used as the positive control. PBS and non-immune serum were used instead of the primary antibody for the blank control and negative control samples, respectively. The slides were visualized with 3, 3'-diaminobenzidine (DAB) detection kit and counterstained with hematoxylin. All the slides were examined and scored independently by two pathologists. ANXA1 protein was stained brown in the cytoplasm with or without in the nucleus.

\section{Cell lines and transfection}

MIA PaCa-2 and BxPC-3 human pancreatic cancer cell lines were purchased from Shanghai GeneChem Co., Ltd. Cells were cultured in DMEM supplemented with $10 \%$ fetal bovine serum (Invitrogen, Shanghai, China).

The pcDNA6.2-GW/EmGFP-miR vector was purchased from Invitrogen (Shanghai, China). The designed oligonucleotides were annealed, followed by ligation into pcDNA6.2-GW/EmGFP-miR vector. Transfection of the control and ANXA1 plasmids into Lipofectamine 2000 reagent (Invitrogen, Shanghai, China) following the manufacturer's instructions. Transfected cells were selected with $10 \mu \mathrm{g} / \mathrm{ml}$ of blasticidin and cloned by limiting dilution. ANXA1 low expression clones were screened for GFP expression by inverted fluorescence microscope. Expression of ANXA1 was confirmed by Western blot method.

\section{PDAC cell proliferation assay}

Cell proliferation was analyzed using a WST-8 Cell Counting Kit-8 (Beyotime, Nantong, China); transfected cells were plated into 96 -well plates at a density of $4 \times 10^{3}$ cells/well. $10 \mu \mathrm{l}$ CCK-8 solution was added to each well and the cultures were incubated at $37^{\circ} \mathrm{C}$ for $1 \mathrm{~h}$. Absorbance at $450 \mathrm{~nm}$ was measured on an ELX-800 spectrometer reader (Bio-Tek Instruments, Winooski, USA).

\section{Cell cycle analysis}

Cell cycle was detected by KeyGEN DNA content Quantitation ASSAY (Cell Cycle) (KeyGEN BioTECH, Nanjing, China). Transfected cells were rinsed with PBS and resuspended $1 \times 10^{6}$ cells in $500 \mu \mathrm{l}$ cold $70 \%$ ethanol and incubated at $4^{\circ} \mathrm{C}$ overnight. After washing with PBS, $100 \mu \mathrm{l}$ Rnase was added to incubation for $30 \mathrm{~min}$ in $37^{\circ} \mathrm{C}$ bath. Then, $400 \mu \mathrm{l}$ PI was added to incubate for $30 \mathrm{~min}$ in dark place. Samples were then analyzed using a FACS Canto flow cytometer (BD Biosciences, Mississauga, Ontario, Canada).

\section{Migration assay}

Cell migration was determined by using a modified two chamber migration assay with a pore size of $8 \mu \mathrm{m}$. $1 \times 10^{6}$ MIA PaCa- 2 cells and $5 \times 10^{5} \mathrm{BxPC}-3$ cells were suspended in $200 \mu \mathrm{l}$ serum-free DMEM medium and seeded into the upper chamber of each insert. Then, $600 \mu \mathrm{l}$ DMEM containing $10 \%$ FBS was added to a 24 -well plate. After $12 \mathrm{~h}$ incubation at $37^{\circ} \mathrm{C}$, cells in the upper chamber were removed by a cotton swab and the cells that had traversed the membrane were fixed in methanol and stained with leucocrystal violet. The number of invasive cells was determined by counting the leucocrystal violet stained cells. For quantification, cells were counted under a microscope in five fields (up, down, median, left and right. $\times 100)$.

\section{Invasion assay}

The invasion assay was performed using a modified two chamber plates with a pore size of $8 \mu \mathrm{m}$. The transwell filter inserts were coated with matrigel (BD Biosciences, NJ, USA). $1 \times 10^{6}$ MIA PaCa- 2 cells and $5 \times 10^{5}$ BxPC-3 cells were suspended in $200 \mu \mathrm{l}$ serum-free DMEM medium and seeded into the upper chamber of each insert. Then, $600 \mu \mathrm{l}$ DMEM containing $10 \%$ FBS was added to a $24-$ well plate. After $24 \mathrm{~h}$ incubation at $37^{\circ} \mathrm{C}$, noninvasive cells in the upper chamber were removed by a cotton swab. Invasive cells at the bottom of the matrigel were fixed in methanol and stained with leucocrystal violet and counted.

Western blot analysis 
Identification of ANXA1 as a Lymphatic Metastasis and Poor Prognostic Factor in Pancreatic Ductal Adenocarcinoma

Proteins were harvested from cancer cells in the plates. Then aliquots of cell extracts were separated on a 10\% SDS-polyacrylamide gel. The proteins were then transferred to nitrocellulose membrane and incubated overnight at $4^{\circ} \mathrm{C}$ with the following antibodies: rabbit antiMMP-9, rabbit anti-TIMP1 (Cell Signaling Technology, Beverly, MA, USA), and mouse anti- $\beta$-actin (Boster Biotechnology, Wuhan, China). Membranes were then washed and incubated with secondary antibody (goat anti-rabbit and goat anti-mouse $\operatorname{IgG}$ ) for $2 \mathrm{~h}$. Membranes were then washed and scanned on the Odyssey TwoColor Infrared Imaging System (LI COR Biotechnology, Lincoln, Nebraska, USA).

\section{Statistical analysis}

SPSS 16.0 statistical software package was used for the statistical analysis. Two-factor analysis of variance procedures and the Dunnett's $t$ test were used to assess differences within treatment groups. Association between ANXA1 expression and the clinicopathological characteristics were analyzed, using Chi-square test. Survival curve was plotted by the Kaplan-Meier method and compared by the log-rank test. Cox's proportional hazards model was used to identify the prognostic factors that influenced survival. $p<0.05$ was considered statistically significant.

\section{Results}

Correlation of ANXA1 expression with clinicopathological parameters

According to immunohistochemistry results we found positive ANXA1 was stained in brown mainly expressed in cytoplasm with or without nuclear staining (Figure 1). Assessment of the association between ANXA1 expression and the clinicopathological data is presented in Table 1. Our data showed that decreased expression of ANXA1 was significantly correlated with poor tumor differentiation

Table 1. Comparison of Clinicopathological Features in Pancreatic Cancer Patients

\begin{tabular}{lllrl}
\hline Variables & \multicolumn{4}{c}{ ANXA1 staining } \\
\cline { 2 - 5 } & Positive (\%) Negative (\%) & Total & P \\
\hline Age & & & & \\
$\quad$ 55 & $80(74.1)$ & $28(25.9)$ & 108 & 0.95 \\
$\quad \leq 55$ & $39(72.2)$ & $15(27.8)$ & 54 & \\
Gender & & & & \\
$\quad$ Male & $68(69.4)$ & $30(30.6)$ & 98 & 0.204 \\
$\quad$ Female & $51(79.7)$ & $13(20.3)$ & 64 & \\
Tumor differentiation & & & & \\
$\quad$ Well & $28(84.8)$ & $5(15.2)$ & 33 & 0.003 \\
$\quad$ Moderate & $81(75.7)$ & $26(24.3)$ & 107 & \\
$\quad$ Poor & $10(45.5)$ & $12(54.5)$ & 22 & \\
Lymph node invasion & & & & \\
$\quad$ No & $78(80.4)$ & $19(19.6)$ & 97 & 0.023 \\
$\quad$ Yes & $41(63.1)$ & $24(36.9)$ & 65 & \\
Nerve invasion & & & & \\
$\quad$ No & $75(74.3)$ & $26(25.7)$ & 101 & 0.91 \\
$\quad$ Yes & $44(72.1)$ & $17(27.9)$ & 61 & \\
TNM & & & & \\
$\quad$ I-II & $82(79.6)$ & $21(20.4)$ & 103 & 0.031 \\
III-IV & $37(62.7)$ & $22(37.3)$ & 59 & \\
\hline
\end{tabular}

( $p=0.003$, Figure $2 \mathrm{~A})$. We also found decreased ANXA1 expression was significantly correlated with lymph node metastasis ( $p=0.023$, Figure 2B). Because TNM stage is an important prognostic marker for patients with PDAC, we studied if ANXA1 expression correlates with TNM stage. We found ANXA1 staining was dramatically decreased in TNM stages III-IV compared with stage

A

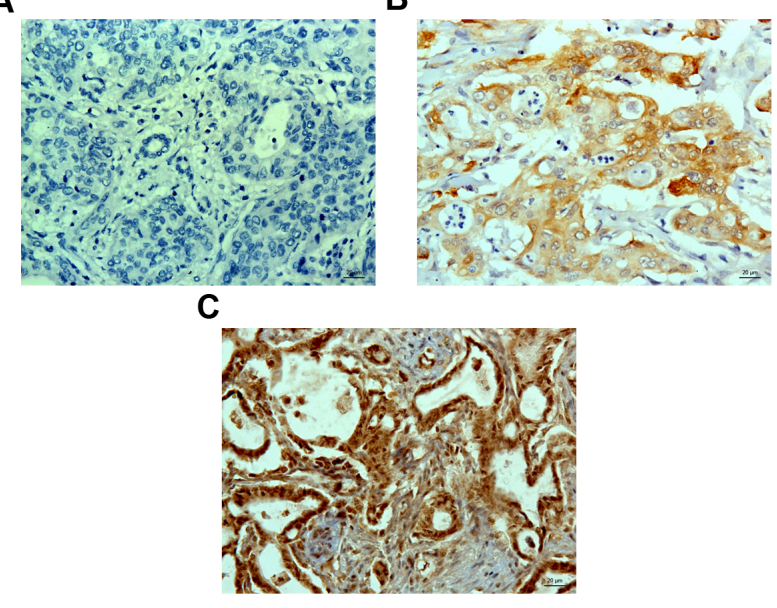

Figure 1. Representative Images of ANXA1 Immunohistochemical Staining in Human PDAC Tissues. A) Negative ANXA1 staining in PDAC. B) Positive ANXA1 was stained in brown mainly expressed in cytoplasm of PDAC. C) Positive ANXA1 was stained in brown mainly expressed in cytoplasm and nuclear of PDAC

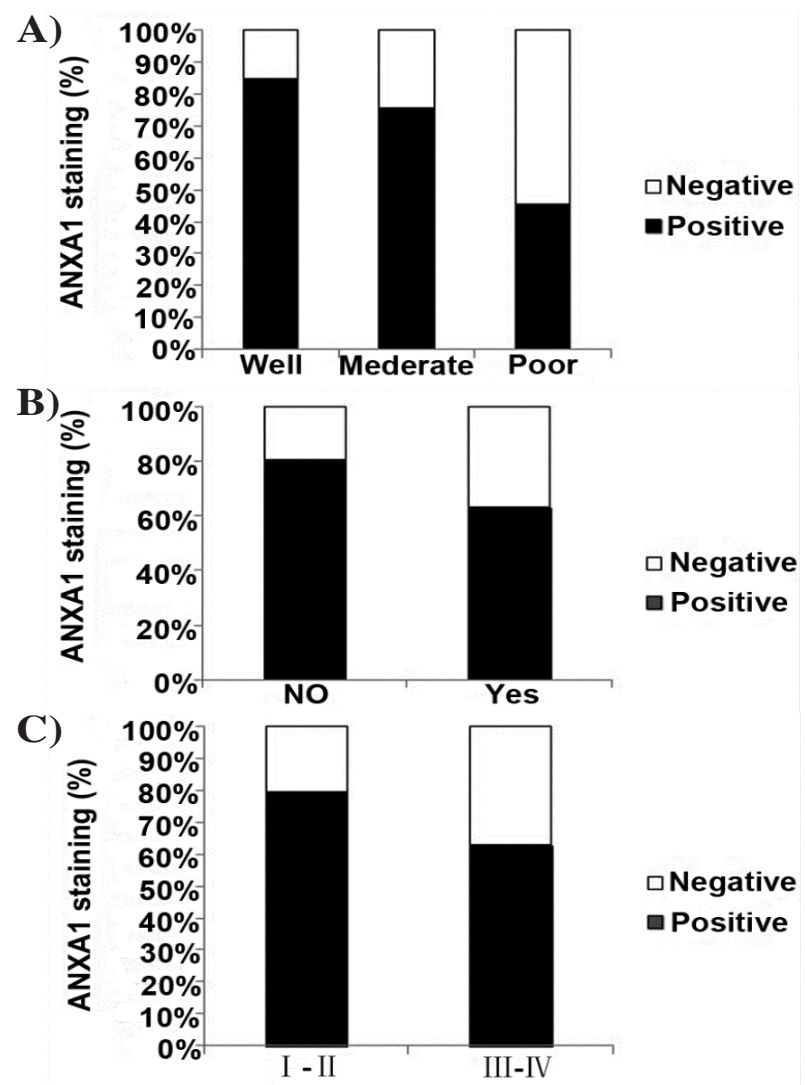

Figure 2. Correlation of ANXA1 Expression with Clinicopathological Parameters. A) Decreased ANXA1 expression was correlated with tumor differentiation $(p=0.003)$. B) Decreased ANXA1 expression was correlated with lymph node invasion $(p=0.023)$. C) Decreased ANXA1 expression was correlated with TNM stage $(p=0.031)$ 
I-II ( $p=0.031$, Figure 2C). However, we did not find significant correlation between ANXA1 expression with other clinicopathologic variables, including age, gender and nerve invasion.

\section{Decreased ANXA1 expression correlates with poor patient} survival

To evaluate whether decreased ANXA1 staining in PDAC correlates with a worse survival of patients, Kaplan-Meier survival curves were constructed to compare the patients with positive ANXA1 staining to those with negative ANXA1 staining. Our data revealed that negative ANXA1 staining correlated with poor prognosis ( $p=0.04$, Figure 3$)$.

Furthermore, we examined whether ANXA1 expression is an independent prognostic marker for PDAC. We performed univariate and multivariate Cox regression analysis including ANXA1 expression, age, gender, tumor differentiation, lymph node invasion, nerve invasion and TNM stage to study the effects of ANXA1

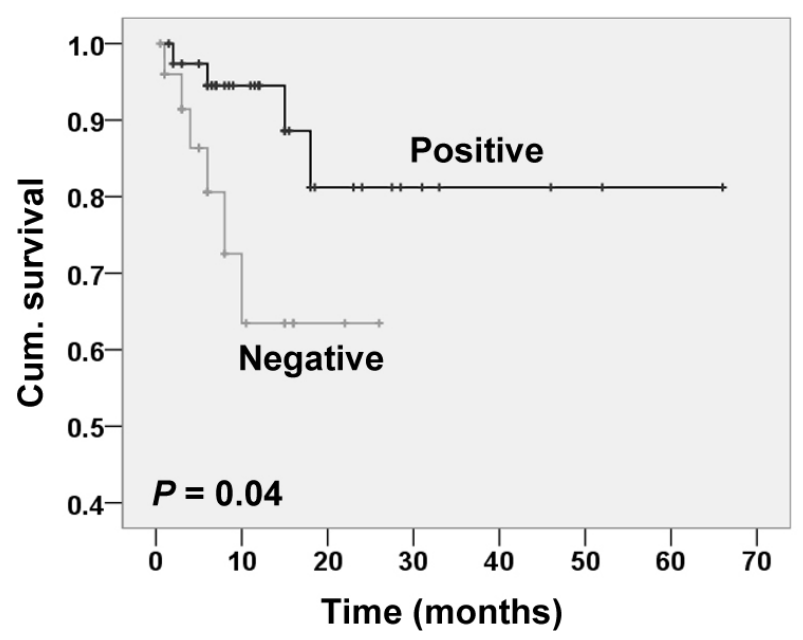

Figure 3. Kaplan-Meier Survival Analysis of PDAC Patients. Decreased ANXA1 expression correlates with a poor survival for PDAC patients ( $p=0.04, \log$ rank test)

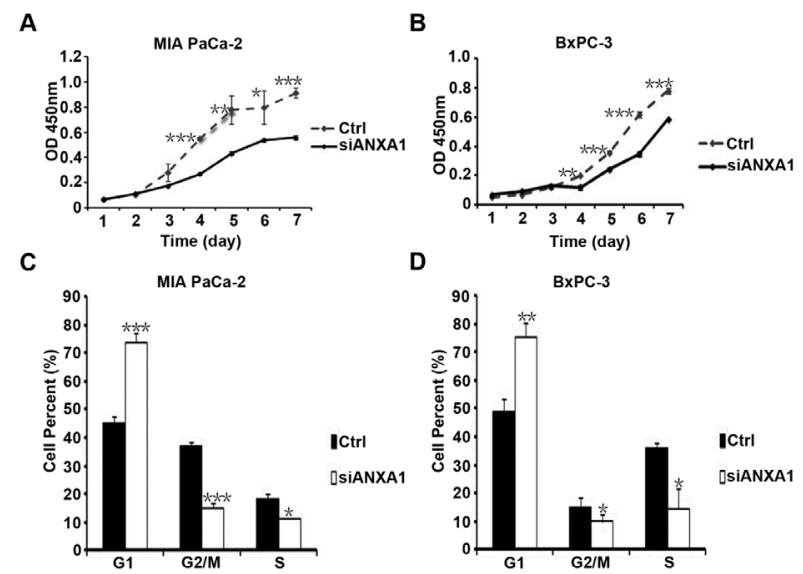

Figure 4. Knockdown of ANXA1 in PDAC Cells Inhibits Cell Proliferation. A, B) CCK- 8 cell proliferation assay after ANXA1 knockdown in MIA PaCa-2 and BxPC-3 cells. C,D) The percentage of $\mathrm{G} 1, \mathrm{G} 2 / \mathrm{M}$ and S population cells was measured by flow cytometry after ANXA1 knockdown in MIA PaCa- 2 and BxPC- 3 cells. $* p<0.05, * * p<0.01, * * * p<0.001$ on patient survival in PDAC. In univariate analysis (Table $2)$, decreased survival was correlated with advanced clinical stage $(p=0.040)$ and decreased ANXA1 expression $(p=0.031)$. In multivariate analysis (Table 3), advanced clinical stage $(p=0.010)$ and decreased ANXA1 expression $(p=0.008)$ remained as the significant independent prognostic factors of decreased survival of patients.

Silencing of ANXA1 in PDAC cell lines inhibits cell proliferation

Because low ANXA1 expression is associated with poor prognosis, ANXA1 may play important roles in one or more steps of tumor metastasis. First, we investigated the involvement of ANXA1 in PDAC cell proliferation. In CCK-8 cell proliferation assay, we found that the ability of cell proliferation was decreased after ANXA1 silence in both MIA PaCa-2 and BxPC-3 cells (Figure 4A, B). To investigate whether the reduced cell proliferation of ANXA1 silence is due to the cell cycle arrest, we performed a flow cytometry analysis. Our data showed that ANXA1 silence cells have significantly higher G1 population than control cells in both cell lines (Figure $4 C, D)$.

Silencing of ANXA1 improves PDAC cells' migration and invasion in vitro

To determine the effect of ANXA1 on PDAC cells migration and invasion, we investigated the migration and invasion of PDAC cells by migration assay and matrigel invasion assay. In cell migration assay, we found that MIA PaCa-2-siANXA1 cells and BxPC-3-siANXA1 cells increase the ability of cell migration (Fig 5A). Meanwhile, the results of cell invasion assay corresponded with the cell migration assay (Figure 5B).

ANXA1 increases MMP-9 expression and activity in PDAC cells

To investigate the mechanisms of ANXA1 regulating migration and invasion, we performed Western blot to

Table 2. Univariate Analysis Of Clinicopathological Factors Affecting Survival of PDAC Patients

\begin{tabular}{lcl}
\hline Variables & \multicolumn{2}{c}{ Univariate analysis } \\
& HR $(95 \% \mathrm{CI})$ & $p$-value \\
\hline Age $(>55 / \leq 55)$ & $1.712(0.958-3.058)$ & 0.069 \\
Gender(male/female) & $0.839(0.480-1.468)$ & 0.539 \\
Tumor differentiation & & \\
Low (reference) & 1 & \\
Moderate & $0.959(0.359-2.559)$ & 0.933 \\
High & $0.535(0.249-1.151)$ & 0.109 \\
Lymph node invasion(yes/no) & $1.037(0.576-1.869)$ & 0.904 \\
Nerve invasion(yes/no) & $1.020(0.569-1.829)$ & 0.947 \\
TNM(I-II/ III-IV) & $0.559(0.321-0.973)$ & 0.04 \\
ANXA1(negative/positive) & $1.897(1.060-3.330)$ & 0.031 \\
\hline
\end{tabular}

Table 3. Multivariate Analysis of Clinicopathological Factors Affecting Survival of PDAC Patients

\begin{tabular}{lcc}
\hline Variables & \multicolumn{2}{c}{ Multivariate analysis } \\
& HR $(95 \% \mathrm{CI})$ & $p$-value \\
\hline TNM(I-II/ III-IV) & $0.366(0.170-0.788)$ & 0.01 \\
ANXA1(negative/positive) & $2.519(1.266-5.012)$ & 0.008 \\
\hline
\end{tabular}


Identification of ANXA1 as a Lymphatic Metastasis and Poor Prognostic Factor in Pancreatic Ductal Adenocarcinoma

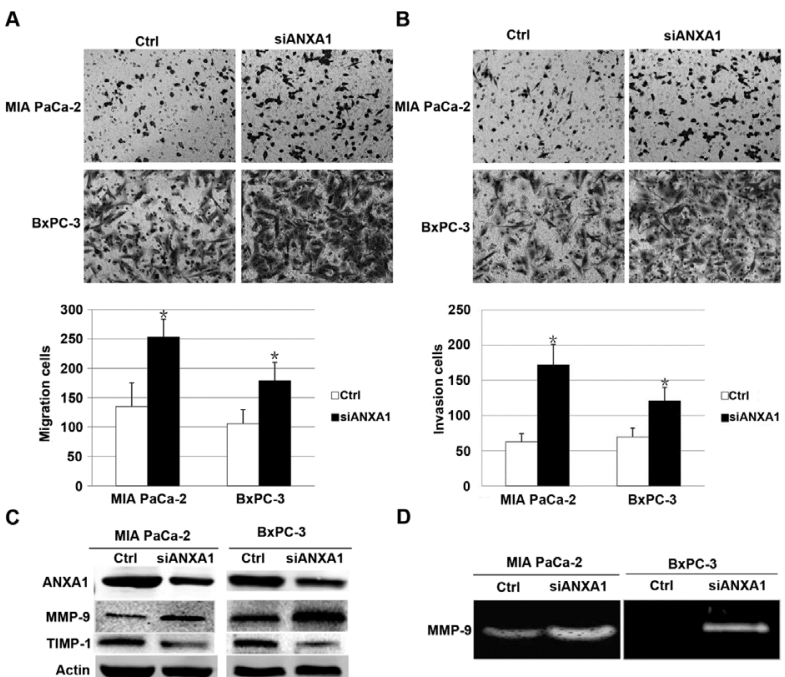

Figure 5. Silencing ANXA1 Increased PDAC Cell Migration and Invasion by Regulating MMP-9 and its Inhibitor TIMP-1. A) Cell migration assay of ANXA1 decreased and control cells. B) Matrigel cell invasion assay of ANXA1 decreased and control cells. $* p<0.05$. C) Western blot analysis of protein levels of MMP-9 and TIMP-1 in ANXA1 decreased and control cells. D Gelatin zymography analysis of MMP-9 in ANXA1 decreased and control cells

detect the MMP-9 and TIMP-1 in PDAC cells. Our data showed that the MMP-9 protein level was increased in MIA PaCa-2-siANXA1 cells and BxPC-3-siANXA1 cells, while the TIMP-1 protein level was decreased (Fig. 5C). Gelatin zymography results showed that MMP-9 enzyme activity was significantly increased in MIA PaCa-2siANXA1 cells and BxPC-3-siANXA1 cells (Figure 5D).

\section{Discussion}

ANXA1 firstly was discovered as a $37-\mathrm{kDa}$ protein and was initially shown to be a glucocorticoid-regulated phopholipase A2 (PLA2) inhibitor (Blackwell et al., 1980). Its physiologic functions have remained largely unclear (Lim and Pervaiz, 2007). ANXA1 may influence cellular behaviors, such as cell proliferation, motility, invasiveness, and cancer-related signaling pathways, which suggest that ANXA1 have important functions in tumor development and progression and can be used as potential biomarkers for the diagnosis and treatment of cancer (Kang et al., 2002; Hongsrichan et al., 2013). Many studies implicated ANXA1 was upregulated with tumor metastasis in mammary tumor, glioma and lung squamous carcinoma progression (Nan et al., 2009; Schittenhelm et al., 2009; Swa et al., 2014). However, downregulated ANXA1 was verified correlating with tumor metastasis too, such as breast carcinoma, gastric cancer and prostate cancer, et al (Cao et al., 2008; Yu et al., 2008; Inokuchi et al., 2009). These results suggest that ANXA1 may play a multifaceted role in tumor development, progression and metastases.

In the present study, we found that decreased expression of ANXA1 was associated with poor postsurgical survival, poor differentiation, lymphatic metastasis and advanced TNM stage of PDAC (in line with earlier findings (Chen et al., 2012).. Moreover, the decreased expression of ANXA1 was an independent prognostic marker of PDAC patients.

Our in vitro studies found that the ability of cell proliferation was drastically decreased after ANXA1 knockdown in PDAC cells, which was due to inhibition of cell-cycle progression by arresting cell cycle at G1 phase. In migration and invasion assay we found that decreased ANXA1 could increase the cell invasion and migration abilities. Our results are consistent with other study in cancer metastatic(Wells et al., 2013), the study found that there's correlation of low proliferative rate in metastatic cancer cells. Furthermore, the conversion of metastatic cells from a mesenchymal phenotype to a more epithelial one also is supportive as these epithelioid tumor cells tend to have a reduced mitogenic rate (Yates et al., 2007; Chao et al., 2010).

MMP-9 is thought to be key enzyme involved in the degradation of type IV collagen, which is a component of the extracellular matrix (Chakraborty et al., 2013). TIMP-1 is one of the endogenous inhibitors of the endopeptidases of the matrix metalloproteinase families, act through the formation of a tight complex with MMP-9 and inhibit the activities of MMP-9 (Basu et al., 2012). Our results showed that decreased ANXA1 in PDAC cells could increase the level and activity of MMP-9 and simultaneously reduced TIMP-1 protein levels. These results indicate that the increase of the migratory and invasive effects of decreased ANXA1 is correlated with modulation of MMP-9 and its inhibitor TIMP-1.

In summary, we demonstrate that decreased expression of ANXA1 expression was associated with poor postsurgical survival, poor differentiation, lymphatic metastasis and advanced TNM stage of PDAC. Moreover, the decreased expression of ANXA1 was an independent prognostic marker of PDAC patients. Decreased expression of ANXA1 inhibits cell proliferation by inducing G1 phase cell cycle arrest and significantly increases PDAC cell migration and invasion through regulating the activities of MMP-9 and its inhibitor TIMP-1. These findings would provide new insights into significance of ANXA1 in migration and invasion of PDAC. However, further studies are required to investigate the mechanism of the role of ANXA1 in PDAC.

\section{Acknowledgements}

This project is supported by grants from 2012 Xuzhou Medical College Dean of special scientific research fund for talents (2012KJZ01), the National Natural Science Foundation of China (No.81472663, 81201636), the Science and Technology Department of Jiangsu Province (No.BK2012139), and China Postdoctoral Science Foundation (No. 2014T70549, 2012M511323).

\section{References}

Basu B, Correa de Sampaio P, Mohammed H, et al (2012). Inhibition of MT1-MMP activity using functional antibody fragments selected against its hemopexin domain. Int $J$ Biochem Cell Biol, 44, 393-403. 
Qing-Hua Liu et al

Blackwell GJ, Carnuccio R, Di Rosa M, et al (1980). Macrocortin: a polypeptide causing the anti-phospholipase effect of glucocorticoids. Nature, 287, 147-9.

Cao Y, Li Y, Edelweiss M, et al (2008). Loss of annexin A1 expression in breast cancer progression. Appl Immunohistochem Mol Morphol, 16, 530-4.

Chakraborty A, Dutta J, Das S, et al (2013). Comparison of ex vivo cultivated human limbal epithelial stem cell viability and proliferation on different substrates. Int Ophthalmol, 33, 665-70.

Chao YL, Shepard CR, Wells A (2010). Breast carcinoma cells re-express E-cadherin during mesenchymal to epithelial reverting transition. Mol Cancer, 9, 179.

Chen CY, Shen JQ, Wang F, Wan R, Wang XP (2012). Prognostic significance of annexin A1 expression in pancreatic ductal adenocarcinoma. Asian Pac J Cancer Prev, 13, 4707-12.

Flower RJ, Blackwell GJ (1979). Anti-inflammatory steroids induce biosynthesis of a phospholipase A2 inhibitor which prevents prostaglandin generation. Nature, 278, 456-9.

Hongsrichan N, Rucksaken R, Chamgramol Y, et al (2013). Annexin A1: A new immunohistological marker of cholangiocarcinoma. World J Gastroenterol, 19, 2456-65.

Hynes RO (2003). Metastatic potential: generic predisposition of the primary tumor or rare, metastatic variants-or both? Cell, 113, 821-3.

Inokuchi J, Lau A, Tyson DR, et al (2009). Loss of annexin A1 disrupts normal prostate glandular structure by inducing autocrine IL-6 signaling. Carcinogenesis, 30, 1082-8.

Kang JS, Calvo BF, Maygarden SJ, et al (2002). Dysregulation of annexin I protein expression in high-grade prostatic intraepithelial neoplasia and prostate cancer. Clin Cancer Res, 8, 117-23.

Keleg S, Buchler P, Ludwig R, et al (2003). Invasion and metastasis in pancreatic cancer. Mol Cancer, 2, 14.

Li Y, Wen T, Zhu M, et al (2013). Glycoproteomic analysis of tissues from patients with colon cancer using lectin microarrays and nanoLC-MS/MS. Mol Biosyst, 9, 1877-87.

Lim LH, Pervaiz S (2007). Annexin 1: the new face of an old molecule. FASEB J, 21, 968-75.

Nan Y, Yang S, Tian Y, et al (2009). Analysis of the expression protein profiles of lung squamous carcinoma cell using shotgun proteomics strategy. Med Oncol, 26, 215-21.

Schittenhelm J, Trautmann K, Tabatabai G, et al (2009). Comparative analysis of annexin-1 in neuroepithelial tumors shows altered expression with the grade of malignancy but is not associated with survival. Mod Pathol, 22, 1600-11.

Siegel R, Naishadham D, Jemal A (2012). Cancer statistics, 2012. CA Cancer J Clin, 62, 10-29.

Swa HL, Shaik AA, Lim LH, et al (2014). Mass spectrometrybased quantitative proteomics and integrative network analysis accentuates modulating roles of Annexin- 1 in mammary tumorigenesis. Proteomics.

Wells A, Griffith L, Wells JZ, et al (2013). The dormancy dilemma: quiescence versus balanced proliferation. Cancer Res, 73, 3811-6.

Yates C, Shepard CR, Papworth G, et al (2007). Novel threedimensional organotypic liver bioreactor to directly visualize early events in metastatic progression. Adv Cancer Res, 97, 225-46.

Yu G, Wang J, Chen Y, et al (2008). Tissue microarray analysis reveals strong clinical evidence for a close association between loss of annexin A1 expression and nodal metastasis in gastric cancer. Clin Exp Metastasis, 25, 695-702.

Zeng GQ, Cheng AL, Tang J, et al (2013). Annexin A1: A new biomarker for predicting nasopharyngeal carcinoma response to radiotherapy. Med Hypotheses, 81, 68-70. 